\title{
Trafic illicite et le dollar du discours
}

Version française

\section{Brian Castro}

\section{(2) OpenEdition Journals}

Édition électronique

URL : http://journals.openedition.org/transtexts/160

DOI : $10.4000 /$ transtexts. 160

ISSN : 2105-2549

Éditeur

Gregory B. Lee

Édition imprimée

Date de publication : 1 mai 2006

Pagination : 14-16

ISSN : 1771-2084

Référence électronique

Brian Castro, «Trafic illicite et le dollar du discours», Transtext(e)s Transcultures 跨文本跨文化 [En

ligne], 1 | 2006, mis en ligne le 13 septembre 2009, consulté le 05 mai 2019. URL : http://

journals.openedition.org/transtexts/160; DOI : 10.4000/transtexts.160 


\section{Trafficking in Dangerous Goods by Brian Castro}

The world did not change on September $11^{\text {th }} 2001$.

What the Western world appeared to do was to simplify discussion to the point where ideas retreated behind more familiar geo-political barricades. A full retreat. What the Western world did was to disdain the foreign, in other words, that very ingredient that possessed an irritant to thought, accelerating time into new particles, which can then be viewed differently ... in other words ... to have an experience. The French word expérience, I think, means both "experience" and "experiment", and it captures the rhetorical imperative of vanguard thinking across barricades.

As a novelist, I have never considered writing historical novels, at least, not those which are constrained by their time. I have never considered a retreat to a so-called "correct" or "universal" representation, but have always had an allegorical projection in mind, to push formal structures into new relevancies. But any newness demands a program of reception which tackles the cultural and political divide. If anything, the ironies of history repeat themselves in the latter's reception and representation. The elision of disciplines, for example, as a way of facilitating the doubling and tripling of thought - the way ideas are intrinsically linguistic - is now quite common in most intellectual discourses. Yet the "rhetoric of rhetoric" has not fully been examined, the way in which rhetoric contradicts reason in translation, or the more explicit way in which different experiences of language and life reform discursive methods. And it has to be experienced, which is one way of speaking about translation.

As l've noted elsewhere, if the 9/11 Commission found that the FBI had failed principally through a lack of imagination by not detecting the plots to demolish the World Trade Center, I would suggest that this failure of imagination stemmed directly from the fact that its members did not read foreign novels with small print runs that challenged their way of thinking. They could not imagine themselves into inhabiting the Other through language. There is a direct link here between an instrumental mentality, a deficient vocabulary, and an inability to inhabit another self. The world's only superpower, making judgments under these conditions, can be hazardous for the rest of us. Indeed, one of Walter Benjamin's great insights was that the self-reflexive obscurity of the aesthetic can help us address ethical as well as political concerns.

It is not only translation which brings with it a betrayal commonly experienced by any writer, but there is greater treason in nationalism, which discourages any idea of risk-taking. We have to ask, behind whose barricades are we retreating ? Innovation bases itself on a knowledge and an absorption of what other people in the world are doing. In music or in the visual arts we automatically engage this larger register. Because literature is tied to language, nations tend to be more possessive of it, and this possession culminates in an innately conservative political and moral agenda. Transnationalism as a field of study can raise the stakes in the arena of reception. If the historian, for instance, is fatally monological, doomed to outcomes, then can studies in diasporic cultures, hybrid existences 
and transnational perceptions refocus attention from a systemic malaise to a reality that exists? The recent London bombings, for example, indicate a blind spot, where the problems of generations bred from disaffection have obviously not been given any prominence.

The possibilities of border-crossing had been expanding, but the barriers have gone up to the extent that whole new filters to cultural transmission are being introduced and we're now in an environment where the key signifiers of particular cultural forms are being radically re-written. Everything and everyone is now seen through the lens of security. In other words, I, as a hybrid writer in more senses than one ... I, as one whose forebears would have been depicted as racially other, subaltern, but not dangerous, is now being asked to consider the role of thought as risk, when once it was play. Cultural transmission has become synonymous with trafficking in dangerous goods, be it ideas, texts or people.

The present moment is probably more critical than at any other time in the recent past for analyzing and theorizing the meanings of cultural exchange. Yet the first point of any exchange is surely a linguistic one. It is why a tri-lingual journal such as this reaches beyond the idea, to the experience itself. In this mis-named "globalized" world, where English is the dollar of discourse, it is a huge irony that English readers only ever get a fraction of what is available. There are whole worlds out there beyond their reckoning, in the past and present, where exceptional works and ideas have emerged and are continually emerging without any notice in English, where rich and subtle discourses are taking place in many other languages, where there are writers who are more driven, more iconoclastic and who have a greater imaginative and experiential reach ... writers whom English readers will never discover, in a world obsessed with one language. We need to hesitate before expression. Learn how to exchange. For whiteness has set the terms for humanity: History, in its terms, has always been the necessity of others.

This fumbling, therefore, with words, this balbutiement of the border-crosser, is something everyone should experience; a Diaspora of thought, a scattering of oneself; dispersing ideas across borders as spores and as hopeful chance; disseminating what is rich with possibilities; seeds ... before the authority of the border steals our truths, turning us into stereotypes out of necessity and fear. It seems that it is a far greater (t)error to be stereotyped as "authentic". Thus, the story of the border-crossing has changed. It has never been more timely, nor more necessary, to be plural, to re-connect the vanguard with the social.

\section{Trafic illicite et le dollar du discours}

Le monde n'a pas changé le 11 septembre 2001.

Tout ce que l'Occident donna l'impression de faire fut de simplifier le débat au point de retrancher les idées en deçà de barricades géopolitiques plus familières. Un repli total. Ce que fit l'Occident fut de mépriser l'étranger, cet ingrédient même qui irrite la pensée, accélérant le temps en de nouvelles particules, et qui peut alors être considéré sous un autre angle...ce qui revient pour ainsi dire à... faire une expérience. Le mot français « expérience » signifie, me semble-t-il, à la fois « expérience » et « expérimentation », et il capture l'impératif rhétorique de la pensée d'avant-garde au delà des barricades. 
En tant que romancier, je n'ai jamais voulu écrire de romans historiques, tout au moins ceux limités par leur période. Je n'ai jamais voulu me réfugier dans une représentation présumée "correcte " ou « universelle », mais j'ai toujours gardé à l'esprit une projection allégorique, afin de repousser les structures formelles vers de nouvelles pertinences. Mais toute nouveauté exige un programme de réception défiant les divisions culturelles et politiques. Les ironies de l'histoire se répètent dans la réception et la représentation de ces dernières. De nos jours, par exemple, l'élision des disciplines afin de faciliter le doublement et le triplement de la pensée - la manière dont les idées sont intrinsèquement linguistiques - est assez courante dans la plupart des discours intellectuels. Pourtant, la « rhétorique de la rhétorique » n'a pas été étudiée à fond, la façon dont la rhétorique contredit la raison en traduction, ou encore la manière plus explicite dont les différentes expériences du langage et de la vie réforment les méthodes discursives. Et il faut en faire l'expérience, ce qui est une des manières de parler de la traduction.

Comme je l'ai déjà mentionné ailleurs, si la Commission du 11 septembre a conclu que la raison principale pour laquelle le FBI n'avait pas pu détecter le complot de destruction du World Trade Center était son manque d'imagination, je dirais que ce manque d'imagination venait du fait que ses membres n'avaient pas coutume de lire de romans étrangers en édition limitée qui missent en question leur manière de penser.

Ils ne pouvaient s'imaginer habitant l'Autre par le langage. II existe un lien direct entre une mentalité instrumentale, un manque de vocabulaire et une inaptitude à habiter un moi autre. Que l'unique superpuissance mondiale rende son jugement dans ces conditions est dangereux pour le reste d'entre nous. En effet, l'une des grandes découvertes de Walter Benjamin était que l'obscurité autoréfléchissante de l'esthétique pouvait nous aider à aborder des préoccupations aussi bien éthiques que politiques.

Ce n'est pas seulement la traduction qui est porteuse d'une trahison expérimentée communément par n'importe quel écrivain, la trahison commise par le nationalisme est bien plus grave qui décourage toute tentative de prise de risque. Derrière les barricades de qui nous retranchons-nous? L'innovation se base sur une connaissance et une absorption de ce que font les autres dans le reste du monde. En musique ou dans les arts plastiques nous adoptons automatiquement ce registre plus vaste. Mais, parce que la littérature est liée au langage, les nations se montrent plus possessives à son égard, et cette possession atteint son apogée dans un projet moral et politique naturellement conservateur. En tant que champ d'études le transnationalisme peut faire augmenter l'enjeu dans l'arène de réception. Si l'historien, par exemple est fatalement monologique et condamné aux résultats, les études de cultures diasporiques, existences hybrides et perceptions transnationales peuvent-elles recentrer notre attention d'un malaise systémique vers une réalité qui existe ? Les récents attentats de Londres, par exemple, indique une zone d'ombre où le malaise et le mécontentement de générations entières n'ont de toute évidence bénéficié d'aucune attention.

Il était devenu de plus en plus facile de franchir les frontières, mais à présent des murs s'y sont élevés de manière à ce que des filtres de transmission culturelle entièrement nouveaux aient été introduits et que nous nous trouvions à présent dans un environnement où les signifiants clés de formes culturelles particulières sont entièrement réécrits.

Dorénavant, tout et tout le monde est perçu à travers l'objectif de la sécurité. En d'autres termes, moi, en tant qu'écrivain plusieurs fois hybride ... moi, dont les ancêtres auraient été décrits comme autres du point de vue de la race, comme subalternes mais non point dangereux, on me demande maintenant de considérer le rôle de la pensée comme risqué, là où elle n'était jadis que jeu. La transmission culturelle est assimilée à un trafic de substances illicites, que ce soit les idées, les 
textes ou les personnes.

Le moment présent est sans doute plus problématique que n'importe quel autre moment du passé récent en ce qui concerne l'analyse et la théorisation des significations des échanges culturels. Et pourtant, l'intérêt premier de tout échange est certainement linguistique. C'est pourquoi la portée d'un journal trilingue comme celui-ci s'étend bien au-delà de l'idée jusqu'à l'expérience même. Dans notre monde qualifié à tort de "globalisé ", où l'anglais est le dollar du discours, il est très ironique que les lecteurs anglais ne disposent que d'une infime partie de ce qui est publié. II existe des mondes entiers dont ils n'ont aucune conscience, dans le passé et à présent, où des idées et des ouvrages exceptionnels ont vu le jour et continuent de voir le jour sans qu'on leur prête la moindre attention en anglais, des mondes où de riches et subtils discours émergent en de nombreux autres langages, où les écrivains sont plus passionnés, plus iconoclastes et dont la portée est beaucoup plus vaste sur les plans de l'imagination et de l'expérience... des écrivains que les lecteurs anglais ne découvriront jamais, dans notre monde obsédé par une seule langue. Nous devons hésiter avant d'exprimer. Apprendre comment échanger. Car le monde blanc a imposé ses conditions à l'humanité : l'Histoire, selon ses conditions, a toujours été le besoin des autres.

Ainsi, cette recherche de mots, ce balbutiement du transfrontalier, est quelque chose dont tout un chacun devrait faire l'expérience : une diaspora de la pensée, un éparpillement de soi ; dispersant les idées par delà les frontières comme autant de spores et de hasards optimistes ; disséminant ce qui est plein de promesses ; des graines ... avant que les autorités frontalières ne volent nos vérités, et ne nous changent en stéréotypes du besoin et de la peur. II me semble que ce soit une bien plus grande ( $t$ )erreur que d'être stéréotypé comme « authentique ». Ainsi l'histoire du franchissement des frontières a-t-elle changé. II n'a jamais été aussi opportun, jamais plus nécessaire d'être pluriel, de raccorder l'avant-garde au social.

\section{危险商品中的交易}

世界并没有在2001年9月11日那一天发生改变。

西方世界曾经想做的似乎是要把讨论简化到这样一种程度, 即各种理念退至更加熟悉 的地缘政治壁垒的背后。一种完全的退却。而西方世界所做的是轻茂异质, 也就是 说, 恰是那些具备思想刺激因素的成分, 它们可以使时代加速走向新的质点, 于是 这些异质可以不同地被认定为, 换句话说, 拥有一种经验。法语中的 “expérience”词, 我认为同时意味着“经验” (experience)与“试验” (experiment)之意, 它越过壁垒 捕获了先锋思想在修辞艺术上的必要之处。

作为小说家, 我从未考虑过要写历史小说, 至少不是那些拘泥于时代的东西。我从 未考虑过退却至一种所谓的 “正确” 或 “普世”的表征, 但在我头脑中总有一种讽 喻性的影射, 把外在的结构推向新的关联事物。但是任何新颖之处都需要一个接纳 系统以便挑战文化与政治的分野。总而言之, 那就是历史的反讽会在后者的接收与 表征当中再现它们自己。举例而言, 作为促进思想双倍或三倍复制的一种途径一 其思想在本质上是语言性的一种方法, 学科的省略在当今大多数知识分子的话语当 中是相当普遍的。然而 “修辞的修辞学” 并未完全得以检验, 如翻译中修辞与理性 相抵触的情形, 或者更为清晰的是, 语言与生活的不同经验革新了推论的方法。这 是必须要体验的, 是谈论翻译的一种途径。 
正如我在其他地方所提到的，如果“9.11”调查委员会发现美国联邦调查局由于缺乏 想象力而基本宣告失败, 没能察觉到要摧毁世贸中心的阴谋的话, 我想要提出的是 这一想象力的失败直接源于这样一种事实, 那就是其成员没有读过那些版本有限的 外国小说，而这些小说对他们的思维方式提出了挑战。他们不能通过语言想象自己 存在于他者之中。这里，在工具性的心理状态、不充足的词汇量与存在于另一个自 我的无能之间有一种直接的链接。在这些条件下做出判断, 世界唯一的超级强权对 于我们之中的其余者来说是危险的。事实上，瓦尔特·本雅明（Walter Benjamin）的远 见卓识之一就是, 他认为审美标准自我反思的模糊性能够帮助我们表达伦理道德与政 治的利害关系。

不仅仅是翻译会随其带来一种任何作者一般都可以体验到的背离，而且在民族主义 之中也有一种更大的叛逆，它阻碍了任何冒险的想法。我们不得不要问，我们正在 退却至谁的壁垒之后? 革新本身基于一种对世界上其他民族正在所作之事的认知与 吸收。在音乐或视觉艺术当中, 我们机械地使用着这种大量的行话术语。因为文学 受限于语言，民族更趋向于拥有它，而且这种拥有在一种天生保守的政治和道德议 事日程中表现为极至。跨国主义作为一个研究领域能够剔除思想接受中的羁绊。例 如, 如果史学家非得持有一元逻辑, 坚信结果, 那么有关离散文化、混杂生活与跨 国感知的研究能够从已不适用的单一系统性走出, 重新将其注意力聚焦至客观存在 的真实吗? 比如说, 近来伦墩的爆炸就表明一个盲点, 其中伴随着不满而成长起来 的几代人的问题已经明显地不再被给予任何关注。

跨越边际的可能性早已日渐提升，但这些障碍已增至这样一种程度，整个新的文化 传输的过滤已经被引入，而且我们现在正处于一种特殊文化形式的关键能指正被激 进地重新书写的环境中。如今每一件事情和每一个人都是从安全的视角来被审视 的。换句话说, 我, 作为一个更多意义上的混杂作家, 一个其祖先可能早被描绘成 人种的他者（是贱民但不危险的）的人，当思想角色一旦成为游戏的时候，我现在 正被要求将之视为危险因素。文化传输已经变成为危险商品交易的同义词，只不过 它是思想、文本或民族。

就文化交流意义的分析与理论化而言，目前现阶段或许比近来过去任何时刻都更为 关键。但是任何交流的首要一点必定是语言性的。这就是为什么这样一本三种语 言的杂志得以超越这一理念而实现其自身的经验。在这个被不当命名的“被全球 化” 的世界中，英语是话语的货币，而英语世界的读者却仅仅获得了所能得到的一 小部分，这是一件极具讽刺之意的事情。世间有些超出他们估计的完整的世界，无 论是过去还是现在，在那里有许多非同寻常的著作和思想曾经出现过，并正继续呈 现着, 但在英语中却未被查觉, 有许多价值巨大且精妙的话语正在许多其他语言中 出现，有许多作家倍受鞭策，更趋于打破旧习，而且他们还有巨大的想象力和不同 凡响的影响……这是些将决不会被英语世界的读者所发现的生活在被一种语言所困 扰的世界中的作家。在措辞之前, 我们需要犹豫一些。要学会如何交流。因为白人 世界已经为人性圈设了框架: 在这种情况下, 历史一直是他者的必需。

因此这种措辞的语言游戏, 跨越边际者的这种结结巴巴（balbutiement），是每一个 人都应当体验的; 这是一种思想的离散，自我的散布; 跨越国界散布思想，如同孢 子, 如同满怀希望的机遇; 散播着充满多种可能性的事物; 如同种子……在国界当 局窃取我们的真理, 把我们转化为失去需求与恐惧的刻板印象之前。被刻板定性 为 “本真的”, 看起来像是一个极大的恐惧或错误。因此跨越国界的故事已经发生 了变化。作为多元的复合体, 把先驱者与社会活动重新联接起来, 显得从未如此及 时过, 也未曾如此必要过。 